\title{
Diseño y aplicación de un programa de seguridad basado en el comportamiento SBC para reducir los incidentes y accidentes en las actividades de perforación e inyección en el dique de arranque, proyecto minero Quellaveco
}

\author{
Design and implementation of a safety program based on SBC \\ behavior to reduce incidents and accidents in drilling and injection \\ activities in the arranque dam, quellaveco mining Project
}

Christ Jesús Barriga Paria ${ }^{1}$, Giuliana Marisol Puma Cruz ${ }^{2}$

Recibido: Julio 2021

Aceptado: Noviembre 2021

\begin{abstract}
Resumen.- La investigación "diseño y aplicación de un programa de seguridad basado en el comportamiento SBC para reducir los incidentes y accidentes en las actividades de perforación e inyección en el dique de arranque, proyecto minero Quellaveco- Moquegua 2020". El objetivo fue diseñar un programa de seguridad basado en el comportamiento para reducir los incidentes y accidentes en las actividades de perforación e inyección en el proyecto minero Quellaveco, empresa contratista SBP S.A.C. La metodología del presente trabajo de investigación es de tipo aplicada tecnológica, se busca reducir los incidentes y accidentes mediante la creación de un programa de seguridad basada en el comportamiento, en razón a conocimientos de la ingeniería de minas, psicología para aplicar en las actividades de perforación e inyección, teniendo un enfoque cuantitativo. Los resultados del diseño y ejecución del programa de seguridad basada en el comportamiento (SBC), demuestra que, reduce los incidentes en las actividades de perforación e inyección y durante el tiempo de la investigación no se reportó ningún accidente. Finalmente se concluye que con la implementación y ejecución del programa se redujo las estadísticas de los incidentes, no se reportó accidentes, mejoro el desempeño en seguridad y promovió la conciencia sobre la seguridad y salud.
\end{abstract}

Palabras clave: accidente fatal; accidente incapacitante; control de riesgo; lechada impermeabilizante; regresión lineal.

Summary.- The investigation "design of a safety program based on SBC behavior to reduce incidents and accidents in drilling and injection activities in the starting dam, Quellaveco-Moquegua 2020 mining project". The objective was to design a behavior-based safety program to reduce incidents and accidents in drilling and injection activities at the Quellaveco mining project, a contractor company SBP S.A.C. The methodology of this research work is of an applied technological type, I seek to reduce incidents and accidents by creating a safety program based on behavior, based on knowledge of mining engineering, psychology to apply in drilling activities and injection, having a quantitative approach. The results of the design and execution of the behaviorbased safety (SBC) program show that it reduces incidents in drilling and injection activities and

1 Ingeniería de Minas, Universidad Nacional de Moquegua (Perú), cbarrigap@ unam.edu.pe, ORCID iD: https://orcid.org/0000-0002-7130-9297

2 Ingeniería de Minas, Universidad Nacional de Moquegua (Perú), pumagiuliana@gmail.com,

ORCID iD: https://orcid.org/0000-0002-5776-4664 
during the time of the investigation no accidents were reported. Finally, it is concluded that with the execution of the program the statistics of incidents were reduced, no accidents were reported, improved safety performance, and promoted awareness about safety and health, generating a new positive attitude on the part of workers.

Keywords: fatal accident; disabling accident; risk control; waterproofing grout; linear regression. 
1. Introducción. - La seguridad basada en el comportamiento es un proceso que busca mediante la observación de comportamientos identificar los actos subestándares, que generen algunas lesiones, las cuales se pretenden prever mediante el programa de seguridad basada en el comportamiento, se realizara planes de acción como retroalimentación, campañas y talleres para reducción de comportamientos inseguros $[1,2,3]$.

Para aplicar el programa de seguridad se hará una colección de datos según factores psicológicos, sociales, institucionales y culturales influyentes en la percepción del riesgo [4], además trabajar más horas puede causar estrés y sobrecargar la vida personal de la persona, lo que podría interferir tanto con los trabajadores como con sus familias [5]. En busca de mantener un ambiente laboral seguro en la industria minera se tiene una serie de protecciones institucionales estandarizadas. [6]. En las empresas mineras tales como minera San Rafael S.A. [7], mina Angélica I, empresa Alma Minerals Perú S.A. [8], contratista IESA S.A en mina Arcata [9], Servicentro Ortiz SRL en mina Antamina [10], Tecniacero S.A.C. de la compañía minera Antapaccay-Cusco [11], J.R. VER S.A.C. en la unidad minera Toquepala [12], la implementación de un programa de seguridad basado en el comportamiento redujo significativamente los actos subestándares.

Este estudio se enfoca en diseñar un programa de seguridad basado en el comportamiento para reducir los incidentes y accidentes en las actividades de perforación e inyección en el proyecto minero Quellaveco, empresa contratista SBP S.A.C. E n este estudio también se determinar la influencia del programa de seguridad basado en el comportamiento para reducir los incidentes y accidentes en las actividades de perforación e inyección, en el proyecto minero Quellaveco, empresa contratista SBP S.A.C.

Esta investigación es fundamental para replicar en otros proyectos, debido a que es una empresa multinacional que construye proyectos de ingeniería civil especializada, infraestructura portuaria, estructuras para sótanos, túneles, obras para el manejo de aguas, construcción de cimentaciones profundas, proyectos de ingeniería geotécnica, anclajes, estabilización de excavadoras, taludes y mejoramiento de suelos.

1.1 Descripción de la problemática. Según la Organización Internacional del Trabajo (OIT) cada año tiene lugar 317 millones de accidentes laborales, de los cuáles 2.3 millones son mortales. El coste de esta adversidad diaria es muy grande y la carga económica de las malas prácticas de seguridad y salud de las empresas suponen un 4 por ciento del producto interior bruto global de cada año [13]. Así mismo, durante el periodo 2009 al 2017 en los 28 países de la Unión europea en la industria minería se produjeron 94,651 accidentes laborales no mortales que produjeron algún tipo de lesión al trabajador y 603 accidentes fatales [14].

En el Perú, según el Ministerio de Energía y Minas (MINEM) [15]., en los últimos 9 años en la industria minera se han registrado en total 840,513 incidentes, 54,921 accidentes leves, 10,978 accidentes incapacitantes, 344 accidentes fatales y 3'080,946 días laborales perdidos. Conforme al último análisis estadístico de accidentes mortales del ministerio de energía y minas en el 2016, se registró un total de 34 accidentes mortales de los cuales 19 se dio por actos subestándar, 8 por condición subestándar, 7 por acto y condición subestándar.

Esta situación se ha visto reflejada en la construcción de la cortina de inyección de la presa de relave se han registrado 56 incidentes y 02 accidentes leves, durante el periodo 2019 a 2020-I, de los cuales un accidente leve corresponde a la actividad de perforación que se realizó con el cambio de mordaza de la perforadora y el otro a inyección, ruptura de packer en el interior del taladro, proyectando fluido de lechada de cemento, ambos según las investigaciones realizadas muestran incidencia en causas inmediatas por actos subestándares. 


\begin{tabular}{llll}
\hline Año & Incidentes & Accidentes Leves & Accidentes incapacitantes \\
\hline $2020-$ I & 19 & 0 & 0 \\
2019 & 37 & 2 & 0 \\
\hline TOTAL & 56 & 2 & 0 \\
\hline
\end{tabular}

Tabla I. Estadística de incidentes y accidentes

Por lo cual en la actualidad la empresa ha optado por implementar una herramienta de gestión, Seguridad Basada en el Comportamiento, que tiene como objetivo brindar a la gerencia y los trabajadores el poder para reducir y prevenir los accidentes en el ambiente de trabajo reduciendo los sesgos cognitivos que se identifican en el ámbito de la gestión de riesgos [16], mejorar el desempeño en seguridad y promover la conciencia sobre la seguridad y salud, generando una nueva actitud positiva por parte de los trabajadores.

En su investigación Mendoza [17] denominada "Gestión de la seguridad basada en el comportamiento", selecciono 30 artículos en idioma inglés y español, por medio de una revisión sistemática exploratoria, en las cuales se demostró que aplicando el programa de seguridad basado en el comportamiento se redujo los índices de siniestralidad en un $80 \%$, además el compromiso de la alta gerencia es fundamental para que el programa funcione, desarrollando así una cultura en seguridad.

1.2 Principios básicos para implementar el programa seguridad basada en el comportamiento. Martin [3], hay 3 principios básicos, que hemos identificado y que creemos tienen un impacto inmediato y significativo para la organización:

1. Incluir la seguridad en los objetivos de la organización, como eje fundamental de cualquier actuación en este sentido.

2. No buscar culpables e ir más allá en el análisis de incidentes y accidentes. Es cierto que la mayoría de los accidentes tienen su origen en un fallo humano, pero lo que hay que preguntarse es por qué ocurre ese fallo y si estamos haciendo todo lo posible para que ese "mal funcionamiento" del sistema no ocurra.

3. Eliminar la palabra castigo del vocabulario preventivo. Si no hay culpables, no tienen sentido las represalias y sí el valor de las soluciones.

Asimismo, se debe tomar en cuenta que para mejorar la aceptación del programa seguridad basada, el diseño de la retroalimentación debe basarse en los deseos de los trabajadores con respecto a cómo se presenta la retroalimentación [18]. Por otro lado, mediante la impartición de enfoques de diseño experimental reflexivos (es decir, con elementos de juegos y aprendizaje inductivo práctico), el rendimiento de la simulación en el caso de conductores se correlaciona fuertemente con el conocimiento del mundo real y la adquisición de habilidades, mejorando su capacidad laboral [19].

1.3 Hypothesis (H1). La aplicación del programa de seguridad basado en el comportamiento reduce los incidentes y accidentes en las actividades de perforación e inyección, durante la construcción del Dique de Arranque del proyecto minero Quellaveco, en la empresa contratista SBP S.AC. 
2. Materiales y métodos. - Tiene un enfoque cuantitativo, utiliza la recolección y el análisis de datos para contestar preguntas de investigación y probar hipótesis establecidas previamente y confía en la medición numérica, el conteo y frecuentemente en el uso de la estadística para establecer con exactitud patrones de comportamiento de una población de acucerdo a Hernández [20].

Para determinar el tamaño de la muestra se utilizo un muestreo no probabilistico segun a criterio, debido a que el tamaño de la muestra es pequeño seleccionando el tamaño de muestra total de $\mathrm{n}=111$, dividida en 03 guardias, el cual conto con 02 trabajadores por guardia como observadores en el programa SBC [21]. Los participantes fueron reclutados del proyecto minero Quellaveco, en la obra perforación e inyecciones de la Cortafuga Final de la construcción de la presa de relave que está ubicado bajo el dique de arranque, realizado por la empresa SBP S.A.C de la obra Perforación e inyecciones de la Cortafuga Final de la construcción de la presa de relave (Plinto Estribo Izquierdo y Plinto Estribo Derecho).

2.1. Instrumento experimental. - El instrumento de medición, utilizado en el estudio de la seguridad basada en el comportamiento, llevada a cabo en empresa SBP S.A.C. en la obra perforación e inyecciones de la Cortafuga Final de la construcción de la presa de relave, fue "Cartilla de observación de comportamiento", que ha permitido realizar un registro sistemático, valido y confiable del comportamiento en las actividades de perforación e inyección, su confiabilidad de determinan con métodos de correlación para este estudio mínimos cuadrados y regresión lineal.

Siendo la corrección conformada por los comportamientos y planes de acciones como variable independiente " $\mathrm{X}$ " y los incidentes y accidentes, como variables dependientes " $\mathrm{Y}$ ", teniendo como resultado una relación inversamente proporción (pendiente negativa).

Para estimar la confiabilidad del instrumento se aplicó sobre los resultados obtenidos de la muestra, el método de correlación por mínimos cuadrados y regresión lineal, en el cual si obtengo 0.25 en la correlación o coeficiente, esto indica baja confiabilidad; si el resultado es 0.50 , la confiabilidad es media o regular. En cambio, si supera el 0.75 es aceptable, y si es mayor a 0.90 es elevada, para tomar muy en cuenta. En donde el coeficiente de correlación es de 0.99, aplicando la medida de estabilidad cada mes.

El método que se aplica en el instrumento para la recolección de datos es la observación y cuestionarios, en la cual el observador registra los comportamientos [22], en la cartilla de comportamientos de las actividades de perforación e inyección, este instrumento está compuesto por varias partes las cuales se describen:

1. Datos del observador y de la persona observada: Nombre del observador, fecha, actividad observada, frente de trabajo, tiempo en el proyecto de la persona observada, edad, turno de la persona observada, día del turno de la persona observada.

2. Comportamiento en las actividades

- Comportamiento en la actividad de perforación

P1 Planificación de la actividad / herramientas de gestión.

$1.1 \mathrm{El}$ trabajador elabora su ATS/ IPERC y luego los hace firmar por el personal competente y autorizado.

1.2 Elabora sus permisos de alto riesgo.

1.3 Elabora los check list correspondientes a la actividad.

$1.4 \mathrm{El}$ trabajador mantiene la difusión de su JSA en el área de trabajo.

P2 EPP

2.1 Usa de forma correcta el casco.

2.2 Usa de forma correcta la protección auditiva (tapones y orejeras).

2.3 Usa y mantiene en buen estado el respirador.

2.4 Usa guantes, guantes antigolpes de acuerdo con la actividad que está realizando y está en buen estado. 
2.5 Usa lentes de seguridad de acuerdo con la actividad que está realizando y está en buen estado (No rayado o roto).

2.6 Usa zapato de seguridad con protección metatarzal.

2.7 Usa arnés y línea de vida en buen estado.

P3 Revisión de equipos y herramientas.

3.1 El trabajador se asegura que el equipo cuente con Kit antiderrame, extintor en buen estado.

3.2 El trabajador inspecciona las guardas y sensores de bloqueo antes de iniciar de iniciar la actividad.

3.3 Herramientas inspeccionadas y almacenamiento correcto.

$\mathrm{P} 4$ revisión de plataforma de trabajo.

4.1 El trabajador señaliza su área de trabajo.

4.2 Realiza la inspección de los accesos, plataforma de trabajo nivelada y el punto a perforar.

P5 Traslado y posicionamiento de la perforadora

5.1 Colocarse en línea de fuego

5.2 El trabajador mantiene las manos en los controles de mando.

P6 instalación de la Compresora para la perforación rojo.

6.1 El trabajador delimitado su área con barras extensibles/cinta de seguridad y barricada

6.2 El trabajador inspecciona las mangueras y se asegura que cuentan antilatigazos o whip check en buen estado.

6.3 El encendido y apagado de la compresora de aire lo realiza un trabajador competente.

P7 Ejecución de la actividad

7.1 El trabajador coloca su tarjeta y candado de bloqueo.

7.2 Línea de fuego.

7.3 Hacen uso del power grip para manipular las tuberías de perforación.

7.4 El trabajador señaliza su área de trabajo.

7.5 Los miembros del trabajo mantienen comunicación efectiva durante la actividad.

7.6 Al levantar cargas se mantiene un peso máximo de $25 \mathrm{~kg}$ por persona.

7.7 El trabajador hace uso de su arnés en pendiente de inclinaciones mayores a $18^{\circ}$.

P8 Orden y limpieza.

8.1 Orden y limpieza antes, durante y después del trabajo.

8.2 Al generar residuos el trabajador realiza la correcta segregación de residuos.

- Comportamiento en la actividad de inyección

I1 Planificación de la actividad / herramientas de gestión.

1.1 El trabajador elabora su ATS/ IPERC y luego los hace firmar por el personal competente y autorizado.

1.2 Elabora sus permisos de alto riesgo.

1.3 Elabora los check list correspondientes a la actividad.

$1.4 \mathrm{El}$ trabajador mantiene la difusión de su JSA en el área de trabajo.

I2 EPP.

2.1 Usa de forma correcta el casco.

2.2 Usa de forma correcta la protección auditiva (tapones y orejeras).

2.3 Usa y mantiene en buen estado el respirador.

2.4 Usa lentes de seguridad google.

2.5 Usa zapato de seguridad con protección metatarzal.

2.6 Usa arnés y línea de vida en buen estado.

2.7 Usa careta facial en buen estado.

I3 Revisión de equipos y herramientas

3.1 La central de inyección cuenta con Kit antiderrame, extintor en buen estado

3.2 Línea de inyección (mangueras), batería de inyección, válvulas, packer, nitrógeno en buen estado. 
3.3 Herramienta inspección y almacenamiento correcto

I4 Ejecución de la actividad

4.1 El trabajador coloca su tarjeta y candado de bloqueo.

4.2 Línea de fuego.

4.3 El trabajador señaliza su área de trabajo.

4.4 Los miembros del trabajo mantienen comunicación efectiva durante la actividad.

4.5 $\mathrm{Al}$ levantar cargas se mantiene un peso máximo de $25 \mathrm{~kg}$ por persona.

4.6 El trabajador hace uso de su arnés en pendiente de inclinación mayor a $18^{\circ}$ (proceso de inyección).

I5. Orden y limpieza.

5.1 Orden y limpieza antes, durante y después del trabajo.

5.2 Almacenamiento correcto de los residuos.

El instrumento presenta la siguiente escala de medición, como se observa en la Figura I.

\begin{tabular}{|l|l|l|l|l|l|l|}
\hline ACT. O. & ITEM & COMPORTAMIENTO & S & I & B & P.E. \\
\hline
\end{tabular}

Figura I. Items de la cartilla de observacion de comportamiento.

Donde:

- ACT. O: Actividad observada.

- ITEM: Numero de los comportamientos.

- Comportamiento: Son los comportamientos observados.

- S: Comportamiento seguro, respuesta representada con un aspa, según el comportamiento observado.

- I: Comportamiento inseguro, respuesta representada con un aspa, según el comportamiento observado.

- B: Barreras, respuesta en escala nominal de 1. Falta de experiencia, 2. Presión de tiempos, 3. Atajos, 4. No hay una sanción, 5. No es cómodo, 6. Olvido, 7. Distraído, 8. Falta de supervisión, 9. No entiendo la indicación dada, 10. Cansancio, 11. Exceso de confianza.

- P. E: Parte expuesta, respuesta en escala nominal de 1. Cabeza, 2. Ojos, 3. Cara, 4. Oídos, 5. Pecho, 6. Brazos, 7. Mano, 8. Piernas, 9. Pies, 10. Cuerpo entero.

Cultura de seguridad salud ocupacional; es un conjunto de valores, principios, normas, costumbres, comportamientos y conocimientos que comparten los miembros de una empresa, para promover un trabajo seguro y saludable, en el que están incluidos el titular de actividad minera, las empresas contratistas mineras, las empresas contratista de actividades conexas y los trabajadores de las antes mencionadas, para la prevención de enfermedades ocupación y daño a las personas.

Para la evaluación de la cultura de seguridad se aplicó una encuesta a 85 trabajadores de la empresa contratista SBP S.A.C., para determinar cual es el nivel de seguridad manejado en la empresa antes de aplicar el programa SBC, formulada en base al Reglamento de Seguridad y Salud ocupación vigente a la fecha D.S. 024-2016 EM y su modificadora D.S. 023-2017-EM, Capítulo I, subcapítulo II, que se detalla en la Tabla II. La encuesta en mencion esta elaborada en la escala de Likert, aplicando el método de alfa de Cronbach [24], tiene una confiabilidad de 0.713, 1.

\begin{tabular}{cc}
\hline Dimensión & Pregunta \\
\hline Valores & ¿Considera que para su empresa la seguridad es un valor \\
& importante? \\
Principios & ¿Considera que la seguridad es un valor importante? \\
¿Se conoce la política integrada de gestión de calidad,
\end{tabular}

Memoria Investigaciones en Ingeniería, núm. 21 (2021). pp. 71-93

https://doi.org/10.36561/ING.21.7

ISSN 2301-1092・ISSN (en línea) 2301-1106 
Normas

Comportamiento

Comportamiento medio ambiente, seguridad y salud ocupacional?

¿Se conoce el reglamento interno de seguridad y salud ocupacional?

¿Le han difundido el procedimiento escrito de trabajo seguro?

¿Se conoce la legislación peruano vigente 29873 ?

¿Realiza un análisis de riesgo antes de iniciar su trabajo?

¿Antes de empezar su trabajo, espera las indicaciones del supervisor o capataz encargado?

¿Hace uso de todos los equipos de protección personal que se le han entregado?

¿Con que frecuencia expone parte de su cuerpo a la línea de fuego, cuando realiza sus actividades?

Tabla II. Ítems de preguntas de la encuesta por dimensiones.

2.2. Variable independiente. - Este estudio empleo un diseño del programa de seguridad basado en el comportamiento, teniendo como indicadores el $n^{\circ}$ de comportamientos proactivos, $n^{\circ}$ de comportamientos reactivos, $\mathrm{n}^{\circ}$ capacitaciones, $\mathrm{n}^{\circ}$ talleres, $\mathrm{n}^{\circ}$ campañas y $\mathrm{n}^{\circ}$ retroalimentaciones.

2.3. Variable dependiente. - La herramienta que se utilizo fue el informe de investigacion detallado por incidente y accidente; ademas se utilizo como indicadores numero de incidentes y accidents por grado de severidad

2.4. Procedimiento. - La confiabilidad de los datos, instrumentos del programa de seguridad basada en el comportamiento en el caso de la encuesta se desarrolló mediante el método de correlación, alfa de Cronbach, calculada a través de la varianza de los datos obtenidos. [23]

Se aplicó el programa estadístico Infostat, regresión lineal por método de mínimos cuadrados para determinar la confiabilidad de los resultados, según Sarabia, J. y Pascual M. [24].

La forma de la función que relaciona las variables X e Y pueden ser de muchos tipos. En primer lugar, consideraremos que el ajuste se realiza mediante una recta con lo que la función será de la forma $\mathrm{y}=\mathrm{a}+\mathrm{bx}$, por tanto, los parámetros a determinar son $\mathrm{a} \mathrm{y} ; \mathrm{b}=0$, se rechaza $\mathrm{H} 0, \mathrm{~b} \neq 0$, se acepta H1 [25].

El ajuste de los mínimos cuadrados consiste en hacer mínima la suma de los cuadrados de las distancias entre los valores observados y los valores ajustados, teóricos o predichos por el modelo de regresión.

Calculamos a y b haciendo mínima la suma de cuadrados de los residuos. La función para minimizar es entonces:

$$
\phi(\mathrm{a}, \mathrm{b})=\sum_{i=1}^{N} e i^{2}=\sum_{i=1}^{N}(y i-y \hat{\imath})^{2}=\sum_{i=1}^{N}(y i-a-b x i)^{2}
$$

Para obtener los valores de a y b que hacen mínima la expresión anterior, tenemos que calcular las derivadas parciales respecto a y $\mathrm{b}$, igualarlas a cero y resolver el sistema. Tenemos que:

$$
\frac{\partial}{\partial \mathrm{a}} \phi(\mathrm{a}, \mathrm{b})=-2 \sum_{i=1}^{N}(\mathrm{yi}-\mathrm{a}-\mathrm{bxi})=0,
$$




$$
\frac{\partial}{\partial \mathrm{b}} \phi(\mathrm{a}, \mathrm{b})=-2 \sum_{i=1}^{N}(\mathrm{yi}-\mathrm{a}-\mathrm{bxi}) \mathrm{xi}=0,
$$

Vamos ahora a probar la descomposicion de la varianza:

$$
\begin{gathered}
S Y 2=\frac{1}{N} \sum_{i=1}^{N}\left[(y i-\hat{y})^{2}\right]=\frac{1}{N} \sum_{i=1}^{N}\left[(y i-\hat{y})^{2}\right] \\
=\frac{1}{N} \sum_{i=1}^{N}(\mathrm{y} \hat{\imath}-\overline{\bar{y}}+\mathrm{ei})^{2} \\
\frac{1}{N} \sum_{i=1}^{N}(y \hat{\imath}-\overline{\bar{y}})^{2}+\frac{1}{N} \sum_{i=1}^{N} e i^{2}+\frac{2}{N} \sum_{i=1}^{N}(y \hat{\imath}-\overline{\bar{y}})^{2} e i^{2} \\
=\mathrm{Sy}^{2}+\mathrm{Se}^{2}+\frac{2}{N} \sum_{i=1}^{N}(\mathrm{y} \hat{\imath}-\overline{\bar{y}}) e i^{2}
\end{gathered}
$$

Veamos que:

$$
\sum_{i=1}^{N}(y \hat{\imath}-\overline{\bar{y}}) e i=0
$$

Efectivamente:

$$
\begin{gathered}
\sum_{i=1}^{N}(y \hat{\imath}-\overline{\bar{y}}) e i=\sum_{i=1}^{N}[a+b x i-(a+b \bar{x})] e i \\
=b \sum_{i=1}^{N}(x i-\bar{x}) e i \\
=b \sum_{i=1}^{N} x i-e i-b \bar{x} \sum_{i=1}^{N} e i=0
\end{gathered}
$$

$\mathrm{Al}$ tener en cuenta las formulas anteriores. Puesto que:

$$
\hat{Y}=a+b x
$$


Se verifica que:

$$
\begin{gathered}
S y^{2}=b^{2} s^{2}{ }_{x} \\
=\frac{S x^{2} y}{S x^{4}} * S X^{2}=\frac{S x y^{2}}{S x^{2} * S y^{2}} * S y^{2}
\end{gathered}
$$

Para probar la ultima propiedad escribimos:

$$
S e^{2}=S y^{2}-S y^{2}
$$

Y hacienda uso de la propiedad anterior:

$$
S e^{2}=S y^{2}-r^{2} S y^{2}=S y^{2}\left(1-r^{2}\right)
$$

Lo que prueba el resultado. Las medidas de ajuste permiten estudiar, la calidad del ajuste de la recta de regresión. Se denomina coeficiente de deteminación de a regression de Y sobre X a:

$$
R y^{2} \mid x=\frac{S y^{2}}{S y^{2}}=1-\frac{S e^{2}}{S y^{2}}
$$

A partir de (6):

$$
R y \mid x 2=x 100
$$

Se puede interpreter como el porcentaje de la variabilidad de la variable dependiente explicada por el modelo de regression.

El coeficiente de determinación verifica las siguientes propiedades:

$$
0 \leq R y^{2}\left|x \leq 1 y-1 \leq R y^{2}\right| x \leq 1
$$

El deficient de determinacion es igual al cuadrado del coeficiente de correlacion lineal:

$$
R y^{2} \mid x=r^{2}
$$

En cuanto a la interpretacion analitica de es preciso analizar los posibles valores de este coeficiente:

- $\quad R_{Y \mid X}^{2}=r=1 \Rightarrow S_{e}^{2}=0$. En este caso; En este caso, diremos que existe correlación perfecta positiva. Es decir, todos los valores teóricos coinciden con los observados y por tanto, la dependencia es funcional. Además, el adjetivo positivo indica que ambas variables varían en el mismo sentido.

- $R_{Y \mid X}^{2}=r=-1 \Rightarrow S_{e}^{2}=0$. La correlación es perfecta negativa. Es decir, la dependencia también es funcional pero las variables varían en sentidos opuestos,

- $\quad R_{Y \mid X}^{2}=r=0 \Rightarrow S_{e}^{2}=S_{y}^{2}$. Las variables no están relacionadas y la correlación es nula. No existe entre las variables una dependencia de tipo lineal. Sin embargo, puede existir otro tipo de dependencia.

- $\quad \mathrm{Si}-1<R_{Y \mid X}^{2}=r<0$. La correlación es negativa y es tanto más intensa cuanto más próxima este

- $\quad 0<R_{Y \mid X}^{2}=r<1$. La correlación es positiva, existiendo mayor relación cuanto más próximo este $R_{Y \mid X}^{2}$ a 1 . 


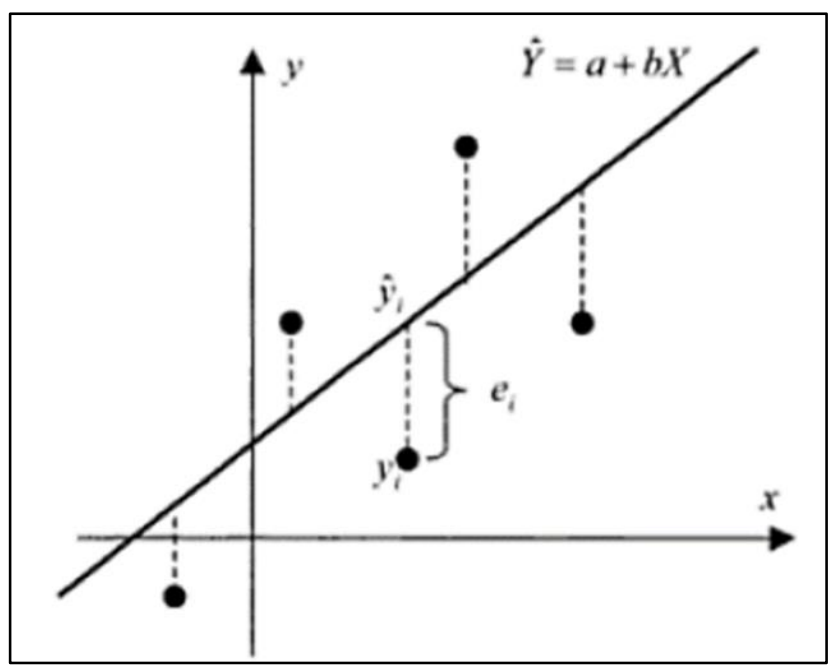

Figura II. Regresión lineal.

3. Resultados. - En los resultados se consideró la información de incidentes y accidentes del proyecto Quellaveco, empresa contratista SBP S.A.C., antes y durante la aplicación del programa así mismo se aplicó exámenes en las capacitaciones, con una calificación máxima de 20 y mínima de 0 para medir el progreso del programa SBC, cada pregunta tiene una valoración en función a este de puede determinar la evoluacion del programa SBC. La presentación de resultados está orientada y organizada estructuralmente según los objetivos específicos y objetivo general planteado en la presente investigación.

3.1. Diseño del programa de seguridad basado en el comportamiento. - El diseño del programa SBC está basado en la observación de los comportamientos (seguros e inseguros) y la ejecución de planes de acciones (capacitaciones, talleres, campañas), para la reducción de incidentes y accidentes en las actividades de perforación e inyección en la empresa SBP SAC, proyecto minero Quellaveco. Los resultados obtenidos se detallan en la Tabla III.

\begin{tabular}{cccccc}
\hline & \multicolumn{2}{c}{ Programa seguridad basada en el comportamiento } & \multicolumn{2}{c}{ Accidentes e incidentes } \\
\hline $\mathrm{N}^{\circ}$ & $\begin{array}{c}\text { Comportamientos } \\
\text { seguros }\end{array}$ & $\begin{array}{c}\text { Comportamientos } \\
\text { inseguros }\end{array}$ & $\begin{array}{c}\text { Capacitaciones } \\
\text { talleres, campañas }\end{array}$ & $\mathrm{N}^{\circ}$ accidentes & $\begin{array}{c}\mathrm{N}^{\circ} \\
\text { incidentes }\end{array}$ \\
$\mathrm{M}$ 1 & 13 & 107 & 1 & 0 & 5 \\
$\mathrm{M} 2$ & 39 & 82 & 1 & 0 & 4 \\
$\mathrm{M} 3$ & 53 & 70 & 2 & 0 & 3 \\
$\mathrm{M} 4$ & 82 & 44 & 2 & 0 & 2 \\
$\mathrm{M} 5$ & 95 & 29 & 3 & 0 & 2 \\
$\mathrm{M} 6$ & 110 & 18 & 3 & 0 & 1 \\
\hline
\end{tabular}

Tabla III. Resumen de los resultados del programa de seguridad basado en el comportamiento 
Comportamientos inseguros y seguros derante la ejecucion del programa SBC en meses.

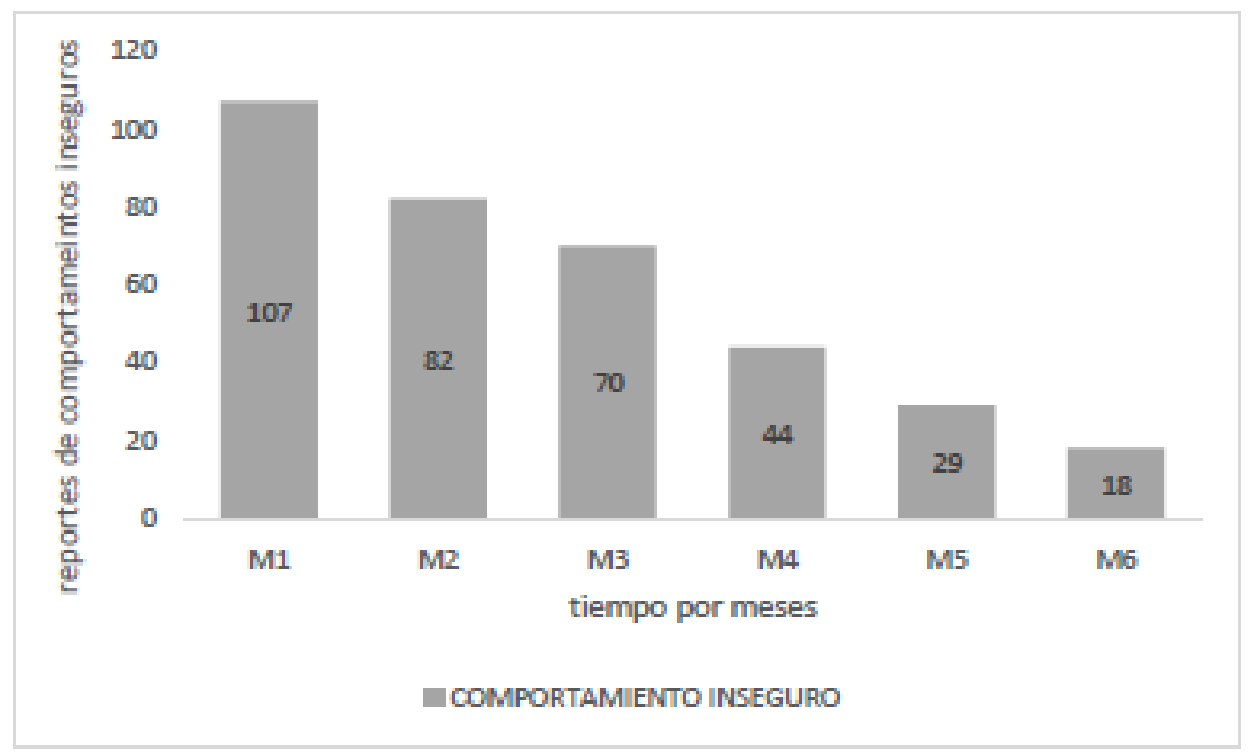

Figura III. Comportamientos inseguros durante la ejecución del programa SBC.

En la Figura III, se observa los comportamientos inseguros, reportados durante los meses de ejecución del programa SBC, que tiene una tendencia decreciente, representando el mayor reporte en el primer trimestre con un total de 259 comportamientos inseguros.

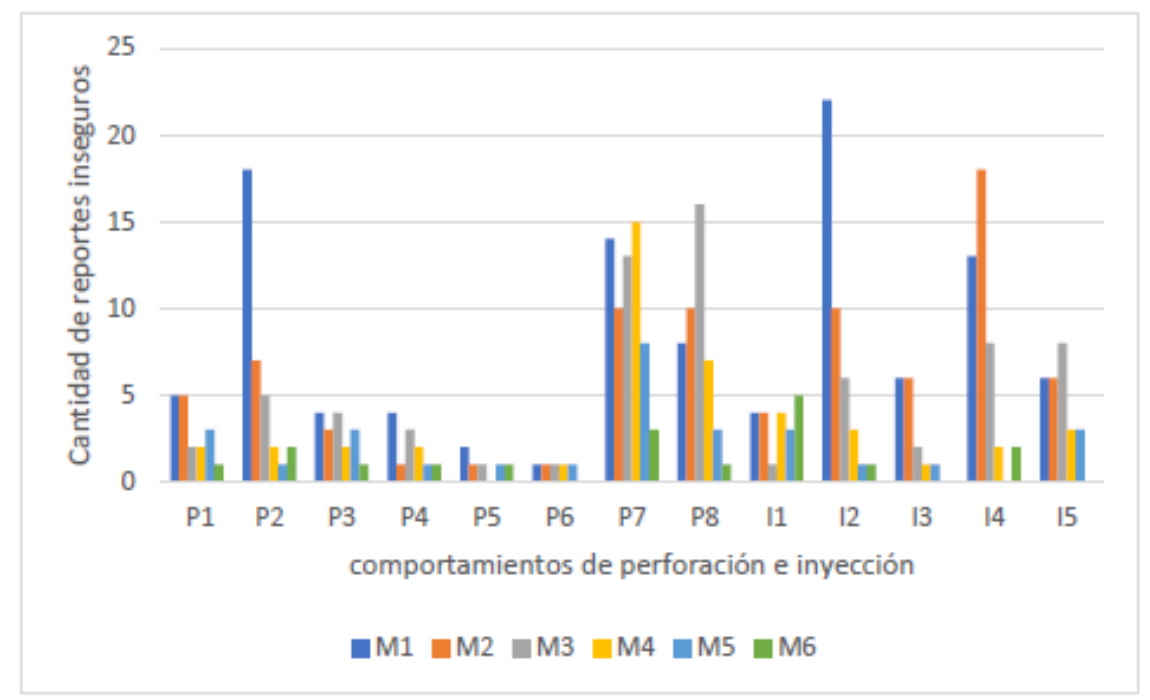

Figura IV. Evolución de comportamientos inseguros observados en 6 meses de aplicación del programa $S B C$.

Memoria Investigaciones en Ingeniería, núm. 21 (2021). pp. 71-93

https://doi.org/10.36561/ING.21.7 
En la Figura IV, se observa la evolución grafica sobre los comportamientos inseguros observados, los cuales destacan por su mayor incidencia:

I2: EPP en la actividad de inyección.

I4: Ejecución de la actividad en la actividad de inyección.

P8: Orden y limpieza en la actividad de perforación.

P7: Ejecución de la actividad en la actividad de perforación

I1: Planificación de la actividad / herramientas de gestión en la actividad de inyección.

Estos resultados indican que los trabajadores de empresa SBP S.A.C. no usan de forma correcta sus equipos de protección personal como casco, respiradores, protección auditiva, lentes de seguridad, guantes, zapatos, metatarzal y arnés en las pendientes mayores a $18^{\circ}$ según los estándares del proyecto minero Quellaveco.

Por otra parte, los trabajadores no cumplen con los estándares del proyecto en la segregación de residuos sólidos como tampoco cuentan con las difusiones actualizadas en sus procedimientos de trabajo seguro (JSA) y en algunos casos se reporta que no cuentan con su procedimiento en campo.

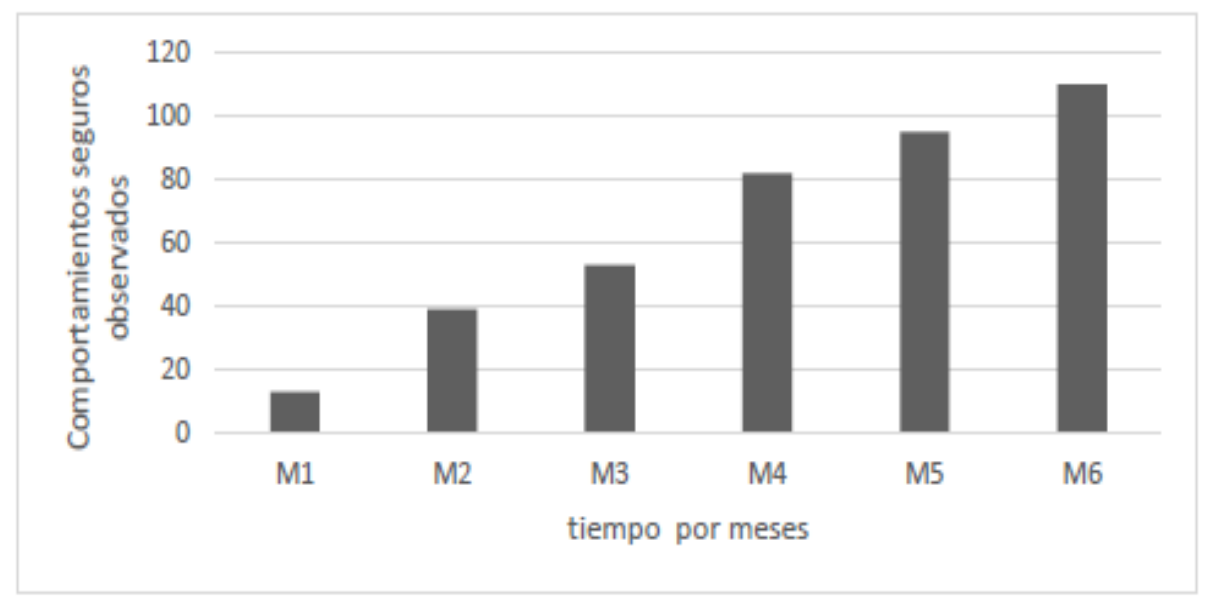

Figura V. Comportamientos inseguros durante la ejecución del programa SBC.

Los comportamientos seguros observados muestran una tendencia creciente como se observa en la Figura V, estos comportamientos aumentan en función del tiempo, como muestra la Figura VI, las cartillas de comportamientos registran que los trabajadores inspeccionan el área de trabajo antes de iniciar sus actividades, inspeccionan sus puntos a perforar, realizan las inspecciones de herramientas y colocan la cinta de mes según estándares del proyecto.

En la Figura VII, podemos observar la evolución del índice de comportamientos, el cual una relación inversamente proporcional, es decir a medida que disminuye el índice de comportamientos inseguros, aumenta el índice de comportamientos seguros, teniendo como resultado durante los 06 meses de ejecución del programa SBC, 47\% en el índice de comportamientos inseguros y 53\% en el índice de comportamientos seguros. 


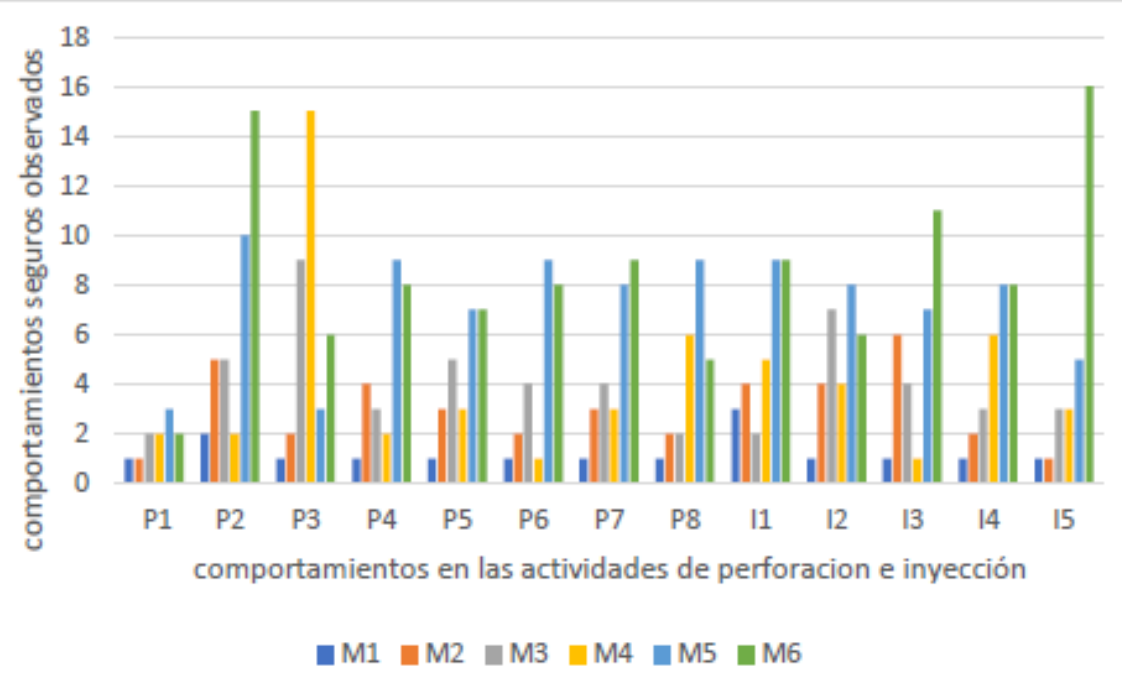

Figura VI. Evolucion de comportamientos seguros observados en 6 meses de aplicación del programa $S B C$.

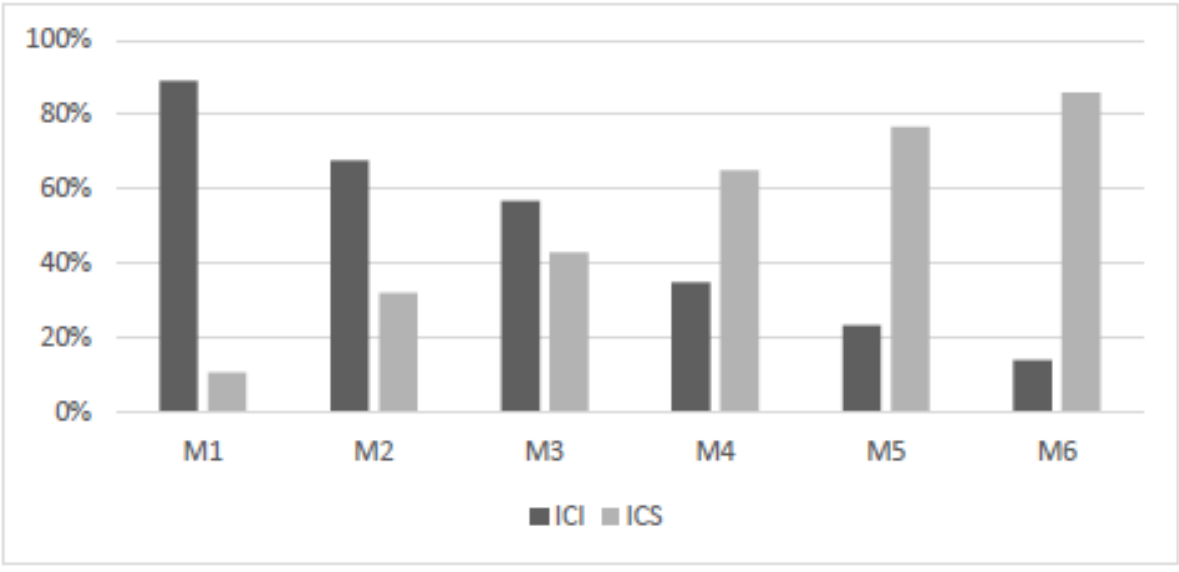

Figura VII. Evolucion del indice de comportamientos (6 meses de aplicación del programa $S B C)$. 
3.2. Indices de seguridad antes y al finalizar el programa SBC. Antes de iniciar el programa de seguridad basado en el comportamiento, se realizó una encuesta, la cual se aplicó a 85 trabajadores de la empresa contratista SBP S.A.C., teniendo como resultado la Figura VIII y Figura IX.

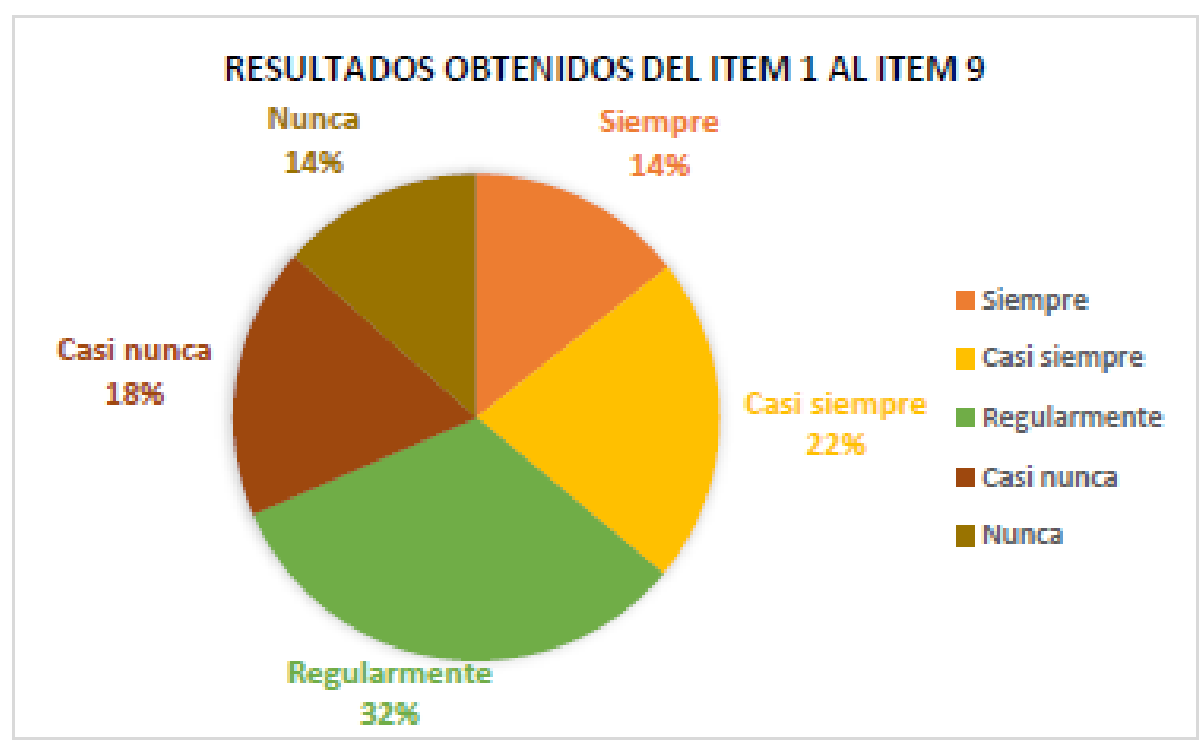

Figura VIII. Resultados obtenidos del ítem 1 al ítem 9 en la primera encuesta.

En la Figura VIII según el contenido de los ítems 1 al 9, están elaboradas para obtener resultados en la clasificación SIEMPRE, la indique todos los trabajadores conocen y practican los valores de seguridad, principios, normas, costumbres y comportamientos seguros. Con los datos obtenidos podemos ver que el mayor porcentaje de respuestas positivas alcanzo la clasificación REGULARMENTE con un $32 \%$.

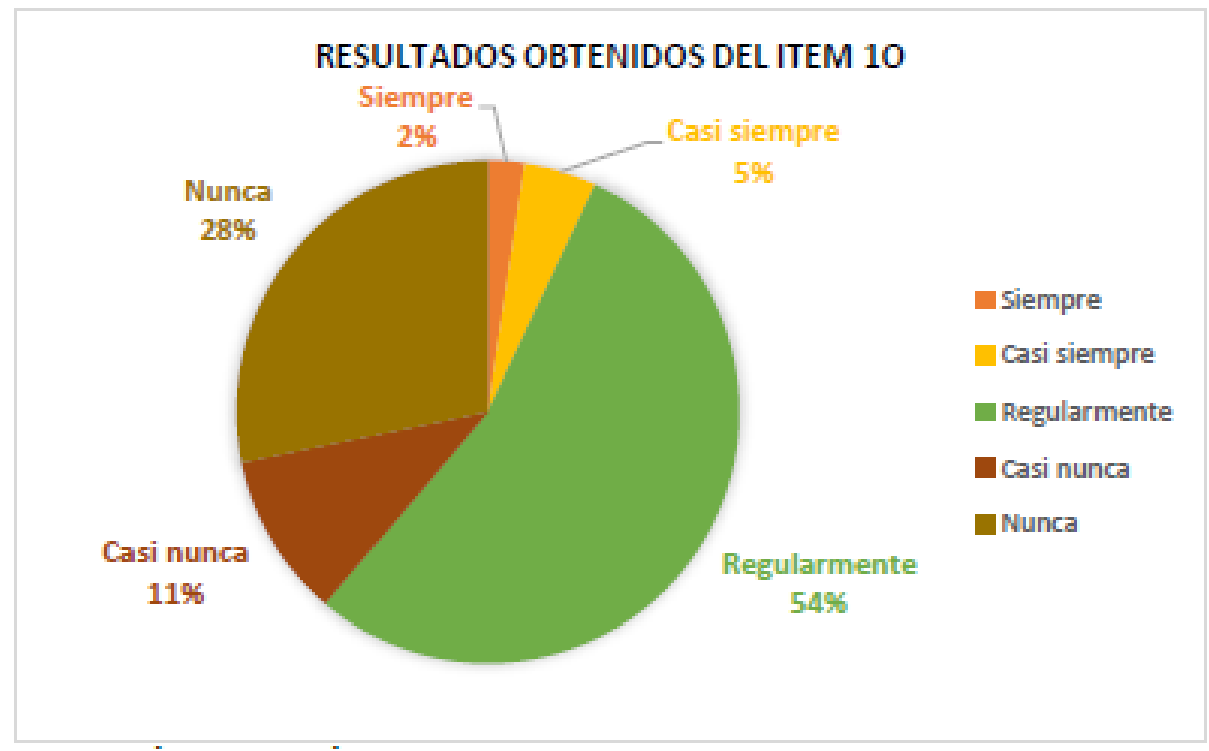

Figura IX. Resultados obtenidos del ítem 10 en la primera encuesta.

Memoria Investigaciones en Ingeniería, núm. 21 (2021). pp. 71-93

https://doi.org/10.36561/ING.21.7

ISSN 2301-1092・ISSN (en línea) 2301-1106 
En la Figura IX, podemos ver el resultado obtenido en la interrogante 10, la cual está elaborada para obtener respuesta en la clasificación NUNCA indicando que los trabajadores no exponen con frecuencia su cuerpo a la línea de fuego, cuando realiza sus actividades, teniendo como resultado un $28 \%$, siendo la clasificación de respuesta REGULARMENTE, el mayor porcentaje representado con un $54 \%$.

Al finalizar el programa de seguridad basado en el comportamiento, se aplicó la misma encuesta, los resultados obtenidos se visualizan en la Figura X y Figura XI.

En la Figura X, se observa que la clasificación de respuesta siempre, ha obtenido el 55\%, seguido de la clasificación casi siempre con un $37 \%$, es decir las variables del programa de seguridad basado en el comportamiento han influido de manera positiva en los cambios de la cultura de seguridad, dado que en los ítems 1,2,3,4,5,6,7,8 y 9, fueron elaborados para obtener respuesta en clasificación siempre, lo que indica que los trabajadores conocen y ponen en práctica comportamientos, valores, principios, normas y costumbres de la seguridad.

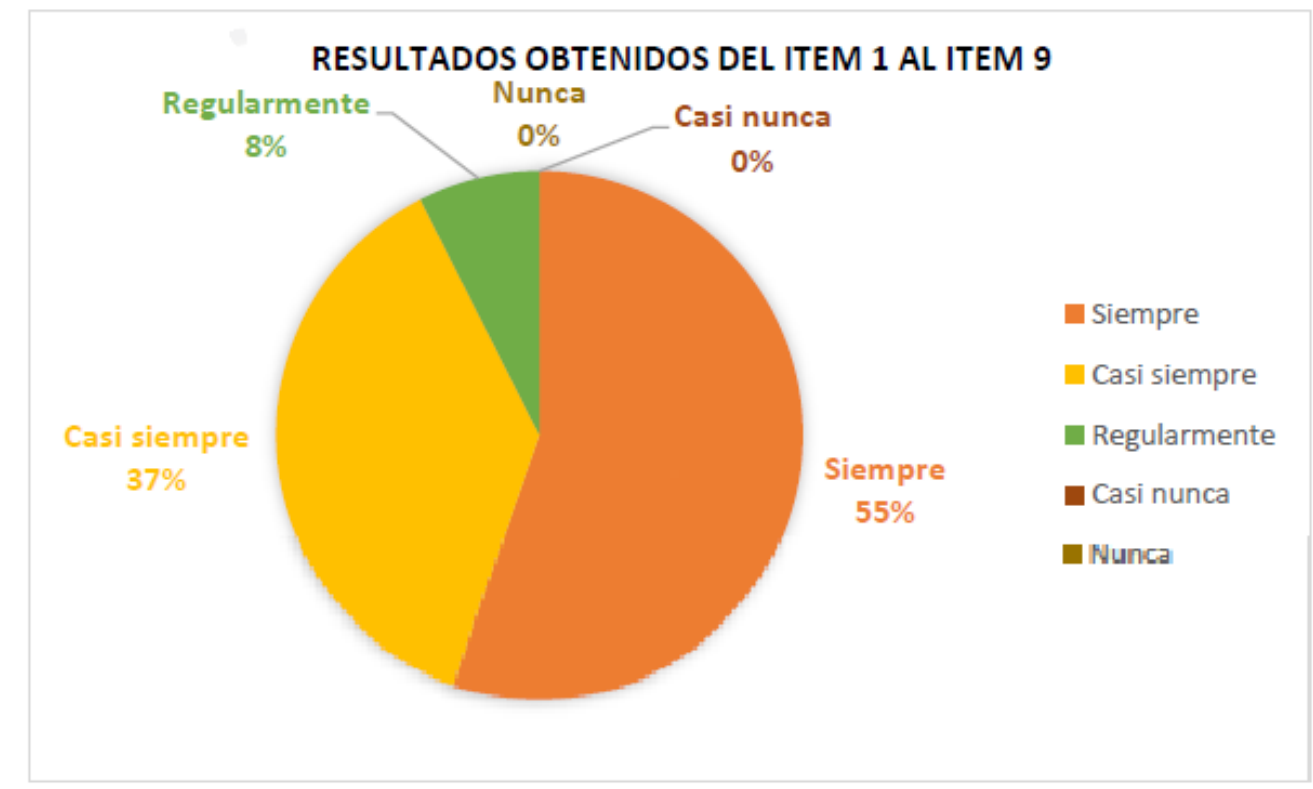

Figura X. Resultados obtenidos del ítem 1 al 9 en la encuesta aplicada al finalizar el programa $S B C$.

En la Figura XI, se observa el ítem 10 de la encuesta aplicada al finalizar el programa de seguridad basado en el comportamiento, tiene como resultado que la clasificación de respuesta NUNCA ha obtenido el $78 \%$, indicando que los trabajadores no exponen las partes de su cuerpo a la línea de fuego en las actividades de su trabajo, lo que indica que el programa de seguridad basado en el comportamiento ha influido de manera positiva en la cultura de seguridad. 


\section{RESULTADOS OBTENIDOS DEL ITEM 10}

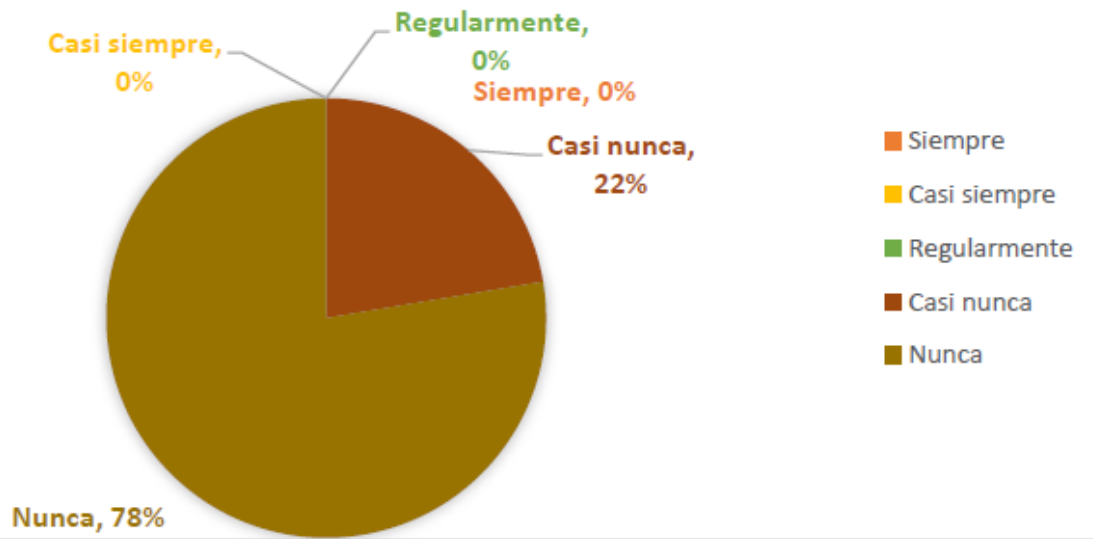

Figura XI. Resultados obtenidos del ítem 10 en la encuesta aplicada al finalizar el programa $S B C$.

Para determinar la influencia de la seguridad basada en el comportamiento para la reducción de accidentes e incidentes, en las actividades de perforación e inyección en la empresa contratista SBP S.A.C. en el proyecto minero Quellaveco, durante el III y IV trimestre del 2020, en la ejecución del programa SBC, se ha elaborado un registro de incidentes y accidentes durante el año 2019 y 2020, los resultados se muestran en la Tabla IV.

\begin{tabular}{lcccc}
\hline Año & Incidentes & $\begin{array}{l}\text { Accidentes } \\
\text { Leves }\end{array}$ & $\begin{array}{l}\text { Accidentes } \\
\text { incapacitantes }\end{array}$ & Accidentes fatales \\
\hline $2020-$ IV & 5 & 0 & 0 & 0 \\
$2020-$ III & 12 & 0 & 0 & 0 \\
2020 -II & 13 & 0 & 0 & 0 \\
$2020-$ I & 19 & 0 & 0 & 0 \\
2019-IV & 18 & 2 & 0 & 0 \\
2019-III & 19 & 0 & 0 & 0 \\
\hline
\end{tabular}

Tabla IV. Registro de incidentes y accidentes por trimestres

3.3. Evalucion de hipotesis, - El presente estudio tuvo como objetivo brindar a la gerencia y los trabajadores el poder para reducir y prevenir los accidentes en el ambiente de trabajo, mejorar el desempeño en seguridad y promover la conciencia sobre la seguridad y salud. Generando una nueva actitud positiva por parte de los trabajadores. Con los resultados obtenidos, se procede a realizar la prueba de la hipótesis general de la investigación donde:

- Hipótesis nula (H0): El diseño del programa seguridad basada en el comportamiento no reduce los incidentes y accidentes en las actividades de perforación e inyección en el proyecto minero Quellaveco, en la empresa contratista SBP S.AC. 
- Hipótesis alternativa (H1): El diseño del programa de seguridad basado en el comportamiento reducirá los incidentes y accidentes en las actividades de perforación e inyección en el proyecto minero Quellaveco, en la empresa contratista SBP S.AC.

4. Discusion de resultados. - El programa seguridad basada en el comportamiento (SBC), está estructurado en comportamientos seguros, comportamientos inseguros, para lo cual aplicaremos la comprobación de la hipótesis en función a cada variable que compone la variable independiente con la variable dependiente los incidentes y accidentes reportados durante los meses de ejecución del programa SBC.

Para la demostración estadística, aplicamos el programa INFOSTAT, por regresión lineal, método de mínimos cuadrados, obteniendo como resultado la Figura XI, en donde se analizó los datos registrados de los comportamientos (seguros e inseguros) con relación a los incidentes y accidentes que se reportaron durante los meses de ejecución del programa.

Siendo en el eje " $X$ " la variable independiente, programa SBC que está conformada por los comportamientos y planes de acción, para lo cual se analizó solo los comportamientos en función a la variable dependiente " $Y$ " incidentes y accidentes, teniendo como resultado una pendiente negativa, es decir cuando la variable $\mathrm{X}$ aumenta la variable $\mathrm{Y}$ disminuye.

Por lo cual existe una relación de disminución de los comportamientos (comportamientos seguros e inseguros reportados en el programa $\mathrm{SBC}$ ) en relación de incidentes y accidentes (R2=0.93; $\mathrm{Y}=61.03+(-0.47) \mathrm{X}$, teniendo un coeficiente de determinación o coeficiente de confiabilidad del $93 \%$, coeficiente de correlación del 0.96 , considerada como una correlación excelente, por consiguiente, se rechaza la hipótesis (H0) y se acepta la hipótesis (H1).

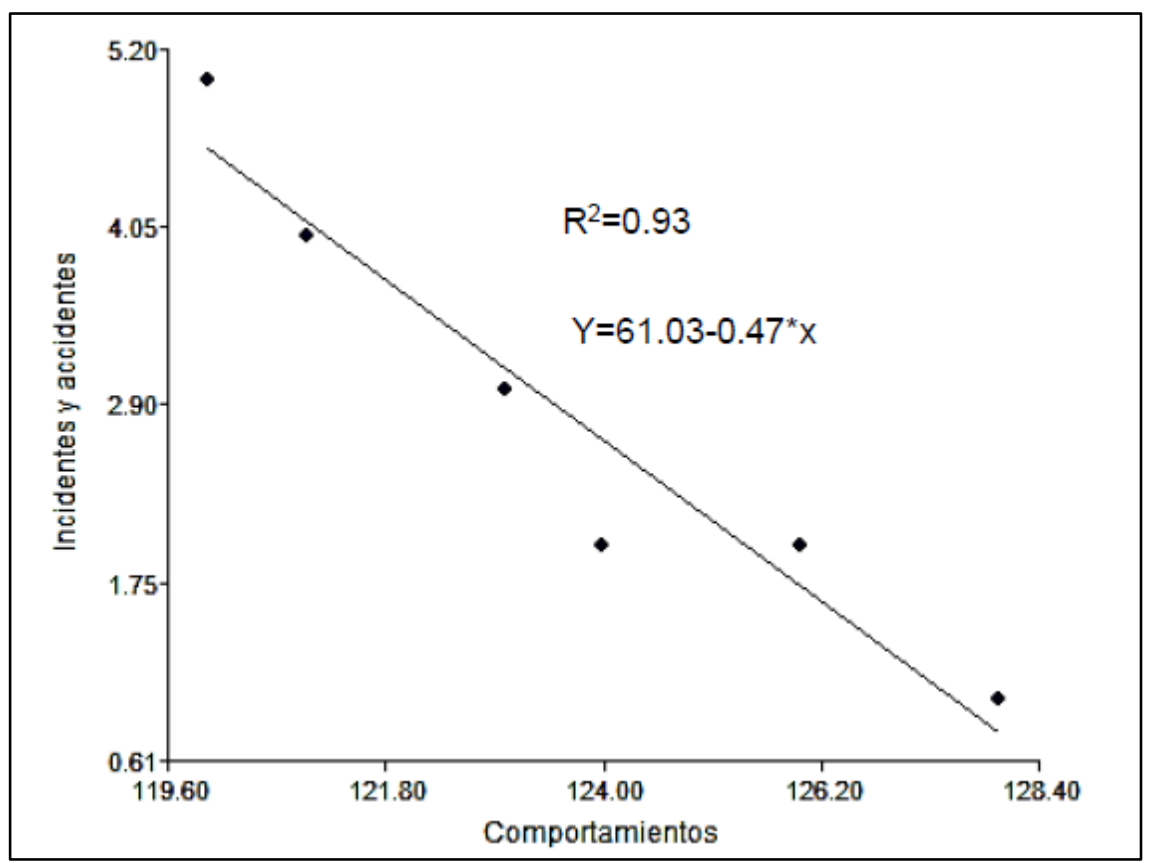

Figura XII. Comportamientos reportados en el programa SBC en función a los incidentes y accidente.

En la Figura XII podemos ver que los resultados de la aplicación de regresión lineal tienen una tendencia creciente, a mayores comportamientos inseguros aumentan los accidentes y accidentes, analizando los datos con el programa INFOSTAT, tenemos una pendiente positiva $(\mathrm{R} 2=0.97$; 
$\mathrm{Y}=0.35+0.04 \mathrm{X})$, teniendo un coeficiente de determinación o coeficiente de confiablidad del $97 \%$ y un coeficiente de correlación de 0.98 , considerada como una correlación excelente.

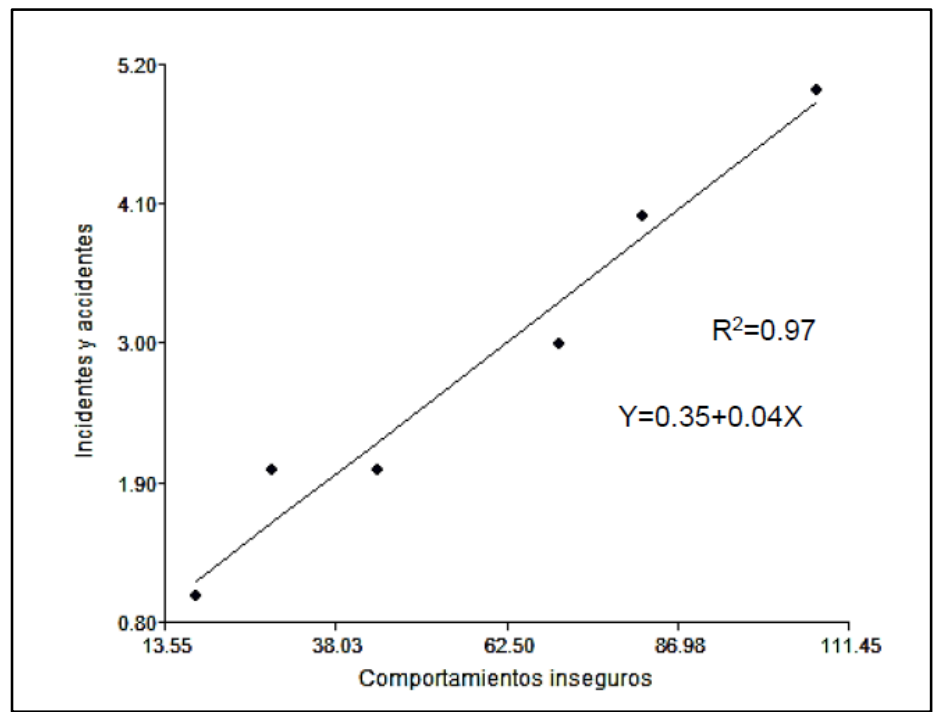

Figura XIII. Comportamientos inseguros en función a los incidentes y accidente.

En la Figura XIV podemos ver una pendiente negativa (R2=0.97; Y=5.42+(-0.04) X), teniendo un coeficiente de determinación o coeficiente de confiablidad del $97 \%$ y un coeficiente de correlación de 0.98 , considerada como una correlación excelente, es decir que a mayores comportamientos seguros disminuyen los incidentes y accidentes.

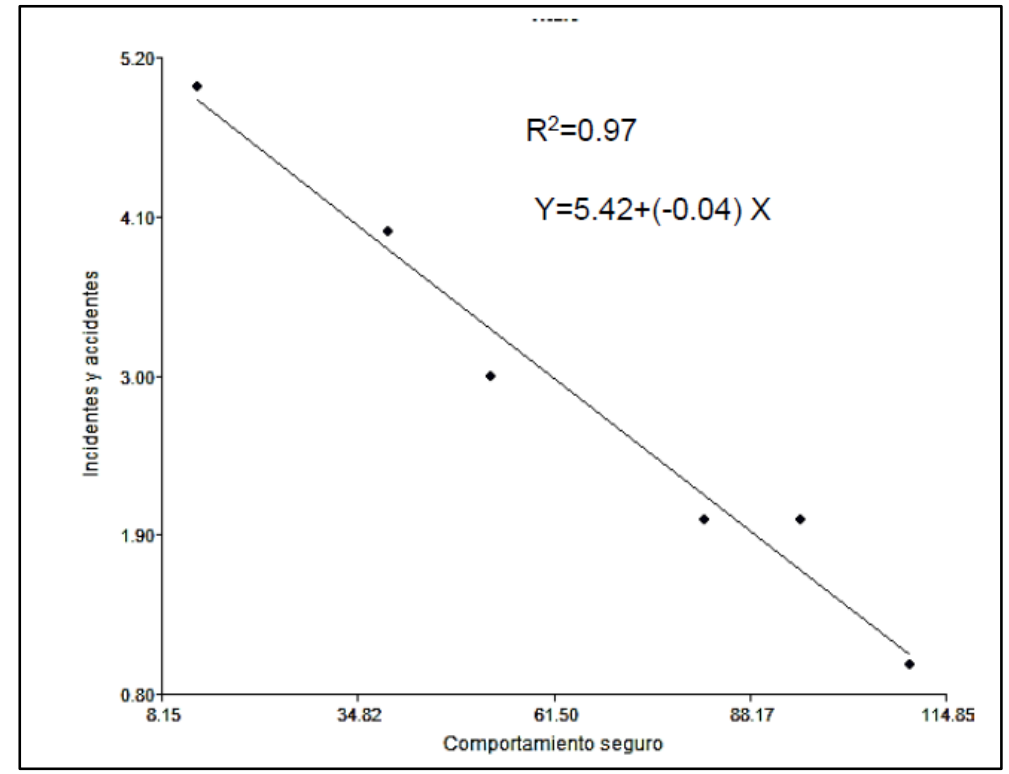

Figura XIV. Comportamientos seguros en función a los incidentes y accidente.

Memoria Investigaciones en Ingeniería, núm. 21 (2021). pp. 71-93

https://doi.org/10.36561/ING.21.7 


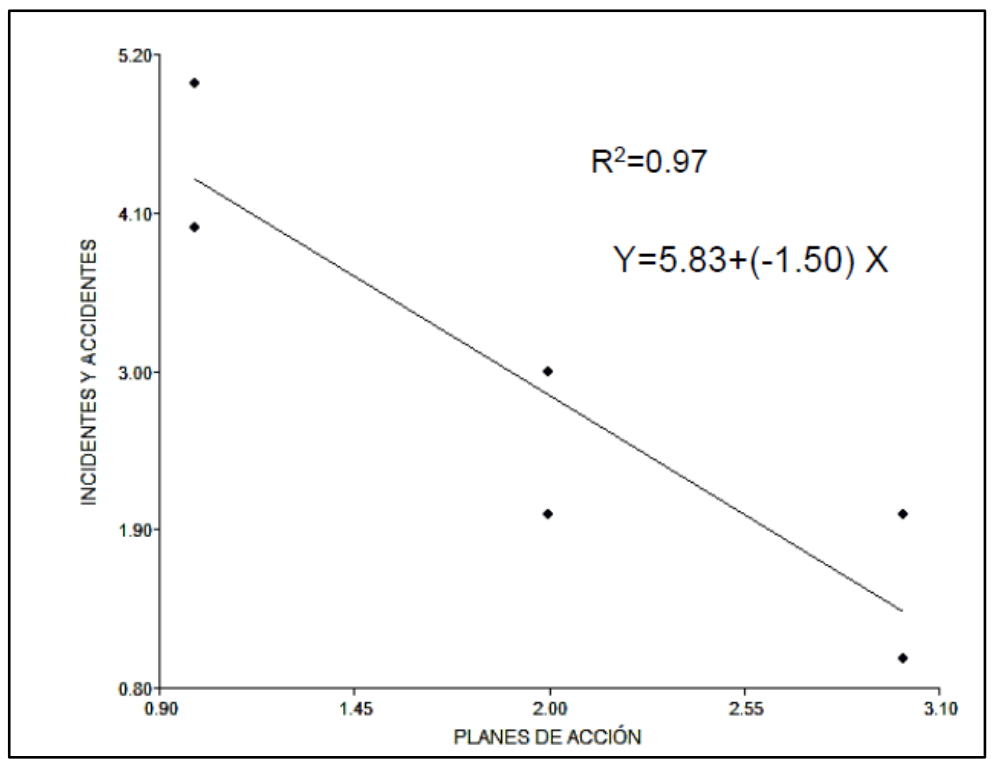

Figura XV. Planes de acción en función a los incidentes y accidente.

En este caso se analiza los datos obtenidos en los planes de acción con relación a los incidentes y accidentes como se observa en Figura XIV la cual aplicando regresión lineal por el método de mínimos cuadrados, se tiene una pendiente negativa $(\mathrm{R} 2=0.97 ; \mathrm{Y}=5.83+(-1.50) \mathrm{X})$, teniendo un coeficiente de determinación o coeficiente de confiablidad del $97 \%$ y un coeficiente de correlación de 0.98 , considerada como una correlación excelente, es decir que a más planes de acción disminuyen los incidentes y accidentes.

\section{Variable independiente $(\mathrm{X}) \quad$ Variable dependiente $(\mathrm{Y}) \quad$ Coeficiente de correlación}

\begin{tabular}{lll}
\hline Comportamiento inseguro & Incidente y accidentes & 0.98 \\
Comportamiento seguro & Incidente y accidentes & 0.98 \\
Planes de acción & Incidente y accidentes & 0.91 \\
\hline Coeficiente correlación promedio & 0.95 \\
\hline
\end{tabular}

Tabla V. Registro de incidentes y accidentes por trimestres. 
5. Conclusiones. - El comportamiento humano representa el mayor porcentaje de causas de los incidentes y accidentes en la empresa contratista SBP S.A.C., en la presente investigación se analizó los resultados obtenidos del diseño y la aplicación del programa de seguridad basado en el comportamiento en las actividades de perforación e inyección, la cual busca que todos los trabajadores puedan, quieran y tengan el conocimiento para poder trabajar seguro, llegando a las siguientes conclusiones:

- Comportamiento en la actividad de inyección En esta tesis el diseñó de un programa de seguridad basado en el comportamiento, redujo los incidentes y accidentes en las actividades de perforación e inyección, analizando las dimensiones de la variable dependiente en relación con la variable independiente se obtuvo un coeficiente promedio de correlación de 0.95. Además, se obtuvo un $47 \%$ en el índice de comportamientos inseguros, $53 \%$ en el índice de comportamiento seguros, indicando una relación inversamente proporcional durante la ejecución del programa.

- El programa de seguridad basado en el comportamiento en las actividades de perforación e inyección influye significativamente en la reducción de accidentes e incidentes, con los análisis estadísticos realizados a la data recopilada de las cartillas de comportamiento, podemos concluir que la confiabilidad promedio es de $92.3 \%$, lo que produce cambios positivos en el comportamiento hacia la seguridad, impactando positivamente las tasas de incidentes y accidentes.

- La ejecución del programa de seguridad basada en el comportamiento redujo durante trimestre 2020-IV y 2020-III, solo el 74\% y $37 \%$ de incidentes en las actividades de perforación e inyección, en la cual no se reportó ningún accidente, en la Plinto Estribo Derecho y Plinto Estribo Izquierdo de la obra perforación e inyecciones de la Cortafuga Final de la construcción de la presa de relave.

\section{Patentes.}

- Fondos: Esta investigación no recibió financiación externa

- Declaración de la Junta de Revisión Institucional: El estudio se realizo de acuerdo con las pautas de la Universidad Nacional de Moquegua, Escuela Profesional de Ingeniería de Minas, ademas agradecer a mis docentes por compartir su experiencia y conocimientos.

- Declaración de consentimiento informado: Se obtuvo el consentimiento informado de todos los sujetos involucrados en el estudio.

- Declaración de disponibilidad de datos: Los datos no están disponibles públicamente debido a la política de protección de datos de los participantes.

7. Agradecimientos. - Los autores desean agradecer sus compañeros de trabajo, Ing. Francis Moreyra, Ing. Luis Mendoza Ureta, Ing. Jenny Guillen Catasi por su orientación, atención a mis consultas y su preciada amistad. Los autores desean agradecer a la empresa Soletanche Bachy Perú (SBP S.A.C), al Ing. Enrique Salcedo, gerente corporativo del área HSE, por tener la disposición en apoyar las investigaciones y brindarme la confianza para realizar la investigación en unos de los proyectos de la empresa. 


\section{Referencias}

[1] Petersen, D. (1997). Behavior-based safety systems: A definition and criteria to assess. Professional Safety.

[2] Martinez Oropesa, C. (2011). El proceso de gestion de la seguridad basado en los comportamientos. El nuevo rol de los supervisors. Universidad Automoma de Occidente.

[3] Martin, M. (2017). Influir en el comportamiento de las personas: Implantación efectiva de programas $\quad S B C$. Prevention World. Disponible: https://preventionworld.com/actualidad/articulos/influir-comportamiento-personas-implantacion-efectivaprogramas-sbc/.

[4] Chaswa, E. N., Kosamu, I. B. M., Kumwenda, S., \& Utembe, W. (2020). Risk Perception and Its Influencing Factors among Construction Workers in Malawi. Safety, 6(2), 12. Disponible: https://doi.org/10.3390/safety6020033.

[5] Smith, T. D., Balogun, A. O., Yu, Z., \& Mullins-Jaime, C. (2020). Health, Physical Activity and Musculoskeletal Symptoms among Stone, Sand, and Gravel Mine Workers: Implications for Enhancing and Sustaining Worker Health and Safety. Seguridad, 6(4). Disponible: https://doi.org/10.3390/safety6040052.

[6] Teja Valluru, C., Rae, A., \& Dekker, S. (2020). Behind Subcontractor Risk: A Multiple Case Study Analysis of Mining and Natural Resources Fatalities. Seguridad, 6(3), 1-18. Disponible: https://doi.org/10.3390/safety6030040.

[7] Guerra Ramirez, J. V. (2017). Programa de seguridad basado en el comportamiento para la minera san rafael, S.A.

[8] Villanueva Chávez, E. J. (2017). Seguridad basada en el comportamiento humano para la prevención de accidentes e incidentes en la mina Maria Angelica I, Empresa ALMA MINERALS PERÚ S.A.

[9] Anibal Sucari, L. (2018). Influencia de la aplicación de seguridad basada en el comportamiento en la ocurrencia de accidentes de trabajo de mina arcata en la empresa contratista IESA S.A. durante el año 2016. Universidad Nacional de Huancavelica.

[10] Huayta Velasquez, N. J. (2018). Implementación de procesos de seguridad basada en el comportamiento para minimizar accidentes en la empresa Servicentro Ortiz Srl Mina Antamina. Universidad Nacional Santiago Antúnez de Mayolo.

[11] Velezmoro Saona, J. L. (2019). Implementación del programa de seguridad basada en la prevención de comportamientos sub estándar en la empresa TECNIACERO SAC. De la compañia ANTAPACCAY-Cusco 2018. Universidad Nacional de Huancavelica.

[12] Faver Mamani, M. M. (2018). Escuela Profesional de Ingeniería de Minas. Universidad Nacional de Moquegua.

[13] Organización Internacional del Trabajo. (n.d.). Retrieved June 3, 2021, from https://www.ilo.org/global/lang--es/index.htm.

[14] Home - Eurostat. (n.d.). Retrieved June 4, 2021, from https://ec.europa.eu/eurostat.

[15] Gobierno del Perú. (n.d.). Retrieved June 3, 2021, from https://www.gob.pe/.

[16] Burggraaf, J., Groeneweg, J., Sillem, S., \& Van Gelder, P. (2019). How Cognitive Biases Influence the Data Verification of Safety Indicators: A Case Study in Rail. Safety, 5(4). https://doi.org/10.3390/safety5040069.

[17] Mendoza Moreira, L. D. (2019). Gestión de la seguridad basada en comportamientos. Universidad San Gregorio de Portoviejo.

[18] Brandhorst, S., \& Kluge, A. (2021). When the Tension Is Rising: A Simulation-Based Study on the Effects of Safety Incentive Programs and Behavior-Based Safety Management. Safety, 7(1). Disponible: https://doi.org/10.3390/safety7010009.

[19] Hulme, K. F., Schiferle, M., Su, R., Lim, A., Estes, A., \& Schmid, M. (2021). Incorporation of Modeling, Simulation, and Game-Based Learning in Engineering Dynamics Education towards 
Improving Vehicle Design and Driver Safety. Disponible: https://doi.org/10.3390/safety7020030. [20] Hernández, S.R., Fernández, C.C., B. P. L. (2004). Metodología de investigación. México. [21] Aldefonso Grande Elena Abascal. (2005). Análisis de encuestas. Disponible: https://books.google.com.pe/books?id=qFczOOiwRSgC\&printsec=frontcover\&hl=es\#v=onepage $\& \mathrm{q} \& \mathrm{f}=$ false.

[22] Nogareda, C., Gracia, D. Á., Martínez, J. F., Peiró, J. M., Lahera, M., \& Meliá, J. L. (2007). Perspectivas de intervención en riegos psicosociales.

[23] Cronbach, L. J. (1951b). Coefficient alpha and the internal structure of tests. Psychometrika, 16(3), 297-334. Disponible: https://doi.org/10.1007/BF02310555.

[24] Pascual Sáez, M., \& Sarabia Alegría, J. M. (2005). Curso básico de estadística para economía y administración de empresas. Disponible: https://www.editorialuc.es/libro/curso-basico-deestadistica-para-economia-y-administracion-de-empresas. 